\title{
Crossing probabilities for critical Bernoulli percolation on slabs
}

\author{
Deepan Basu ${ }^{\mathrm{a}}$ and Artem Sapozhnikov ${ }^{\mathrm{b}}$ \\ ${ }^{a}$ Max-Planck Institute for Mathematics in the Sciences, Inselstrasse 22, 04103 Leipzig, Germany. E-mail: deepan.basu@mis.mpg.de \\ ${ }^{\mathrm{b}}$ Department of Mathematics, University of Leipzig, Augustusplatz.10,04109 Leipzig, Germany. E-mail: artem.sapozhnikov@math.uni-leipzig.de
}

Received 16 December 2015; revised 16 June 2016; accepted 28 June 2016

\begin{abstract}
We prove that in the critical Bernoulli percolation on graphs $\mathbb{Z}^{2} \times\{0, \ldots, k-1\}^{d-2}$, for each $\rho>0$, the probability of open left-right crossing of rectangle $[0, \rho N] \times[0, N] \times[0, k-1]^{d-2}$ is uniformly positive.

Résumé. On démontre que dans la percolation de Bernoulli critique sur le graphe $\mathbb{Z}^{2} \times\{0, \ldots, k-1\}^{d-2}$, pour chaque $\rho>0$, la probabilité d'avoir un passage de gauche à droite ouvert dans $[0, \rho N] \times[0, N] \times[0, k-1]^{d-2}$ est uniformément positive.
\end{abstract}

MSC: 60K35; 82B43

Keywords: Critical Bernoulli percolation; Slab; Russo-Seymour-Welsh theorem

\section{Introduction}

One of the main tools in the study of planar percolation models at criticality is the Russo-Seymour-Welsh (RSW) theorem. It states that the probability that an open path connects the left and right sides of a rectangle is bounded away from 0 and 1 by constants that only depend on the aspect ratio of the rectangle. This theorem was first proved for critical Bernoulli percolation on planar lattices in [7,9-11] and recently has been extended to some other planar models, perhaps most notably to the FK-percolation $[3,4]$ and Voronoi percolation $[2,12]$.

In this note we consider critical Bernoulli percolation on two dimensional slabs $\mathbb{Z}^{2} \times\{0, \ldots, k-1\}^{d-2}$. We prove that the probability of crossing a rectangle is bounded from below by a positive constant which only depends on the aspect ratio of the rectangle and the slab parameter $k$, but does not depend on the size of the rectangle. Our work is inspired by a recent paper of Duminil-Copin, Sidoravicius, and Tassion [5] where they prove the absence of percolation at criticality for slabs and develop techniques for "glueing" open paths. Our proof is partly based on these new ideas.

\section{Notation and result}

Fix an integer $k \geq 1$, and define the slab of width $k$ by

$$
\mathbb{S}=\mathbb{Z}^{2} \times\{0, \ldots, k-1\}^{d-2} .
$$

We consider Bernoulli bond percolation on $\mathbb{S}$ with parameter $p \in[0,1]$, and denote the corresponding measure by $\mathbb{P}_{p}$. Let $p_{c}$ be the critical threshold for percolation, i.e.,

$$
\left.p_{c}=\inf \left\{p: \mathbb{P}_{p} \text { [open connected component of } 0 \text { in } \mathbb{S} \text { is infinite }\right]>0\right\},
$$

and define the measure $\mathbb{P}=\mathbb{P}_{p_{c}}$. 
For a subset $A$ of vertices of $\mathbb{Z}^{2}$, let

$$
\bar{A}=A \times\{0, \ldots, k-1\}^{d-2} .
$$

Define a rectangle and its left and right boundary regions by

$$
B(m, n)=\overline{[0, m) \times[0, n)}, \quad L(m, n)=\overline{\{0\} \times[0, n)}, \quad R(m, n)=\overline{\{m-1\} \times[0, n)} .
$$

Consider the crossing event

$$
\mathrm{LR}(m, n)=\{L(m, n) \text { is connected to } R(m, n) \text { by an open path in } B(m, n)\}
$$

and the crossing probability

$$
p(m, n)=\mathbb{P}[\operatorname{LR}(m, n)] .
$$

In this note we prove the following theorem.

Theorem 2.1. For any $\rho \in(0, \infty)$,

$$
\liminf _{n \rightarrow \infty} p(\lfloor\rho n\rfloor, n)>0 .
$$

Proof. The case $k=1$ is classical; see e.g. [7,10,11]. As some of the "glueing" ideas used in the proof are unnecessary for $k=1$ and easier for $k \geq 2$, we assume from now on without further mentioning that $k \geq 2$. The theorem is proved in 3 steps:

- The result holds for all $\rho \in(0,1)$. This is well known. We give a proof in Proposition 3.1 .

- If the result holds for some $\rho>1$, then it holds for all $\rho>1$. This is a well known fact in planar percolation. We prove the slab version in Proposition 5.1 using the planar approach together with a novel technique for glueing paths from [5] (see Lemma 4.2).

- There exist $c>0$ and $C<\infty$ such that for all $n \geq 1, p(44 n, 43 n) \geq c \cdot p(43 n, 44 n)^{C}$. This inequality is the main contribution of this paper. We prove it in Proposition 6.1 using various "paths glueing" procedures.

Remark 2.2. For $\rho<1$, the result of Theorem 2.1 holds in any dimension $d \geq 2$. We believe that it also holds for $\rho \geq 1$, but do not know a proof. Our method unfortunately crucially relies on planarity of slabs. If dimension is sufficently high, it is proved in [1] that the crossing probabilities tend to 1 as $n \rightarrow \infty$. We believe that for percolation on slabs (and in low dimensions) for every $\rho>0$, $\lim _{\sup _{n \rightarrow \infty}} p(\lfloor\rho n\rfloor, n)<1$, but do not have a proof yet. ${ }^{1}$

Earlier we defined $\bar{A}$ as a subset of $\mathbb{S}$ for each $A \subset \mathbb{Z}^{2}$. In the proofs we will often use the same notation $\bar{A}$ for $A \subset \mathbb{S}$ meaning

$$
\bar{A}=\left\{z=\left(z_{1}, \ldots, z_{d}\right) \in \mathbb{S}:\left(z_{1}, z_{2}, x_{3}, \ldots, x_{d}\right) \in A \text { for some } x_{3}, \ldots, x_{d}\right\} .
$$

This way, for each $A \subset \mathbb{Z}^{2}, \bar{A}$ defined earlier is the same as $\overline{A \times\{0\}^{d-2}}$ defined just above.

\section{Crossings of narrow rectangles}

The following proposition is an adaptation to slabs of a well known fact about the probabilities of crossing hypercubes of fixed aspect ratio in the easy direction.

Proposition 3.1. For any $\rho \in(0,1)$, (2.1) holds.

\footnotetext{
${ }^{1}$ For slabs, this was recently proved in [8].
} 
Proof. Let $0<a<b$ be integers. It suffices to prove that $\liminf _{n \rightarrow \infty} p(a n, b n)>0$. A standard recursive inequality gives that for $C=2\left\lceil\frac{b}{b-a}\right\rceil+1$, every $p \in[0,1]$ and $n \geq 1$,

$$
\mathbb{P}_{p}[\operatorname{LR}(2 a n, 2 b n)] \leq\left(C \mathbb{P}_{p}[\operatorname{LR}(a n, b n)]\right)^{2} .
$$

Indeed, any open left-right crossing of $\overline{[0,2 a n) \times[0,2 b n)}$ produces open left-right crossings of $\overline{[0, a n) \times[0,2 b n)}$ and $\overline{[a n, 2 a n) \times[0,2 b n)}$ giving $\mathbb{P}_{p}[\operatorname{LR}(2 a n, 2 b n)] \leq \mathbb{P}_{p}[\operatorname{LR}(a n, 2 b n)]^{2}$. Then, any open left-right crossing of $\overline{[0, a n) \times[0,2 b n)}$ either crosses horizontally one of the rectangles $\overline{[0, a n) \times[k n,(k+b) n)}, k \in\{0, b-a$, $\left.2(b-a), \ldots,\left\lceil\frac{b}{b-a}\right\rceil(b-a)\right\}$, or crosses vertically one of the rectangles $\overline{[0, b n) \times[k n,(k+a) n)}, k \in\{b-a$, $\left.2(b-a), \ldots,\left\lceil\frac{b}{b-a}\right\rceil(b-a)\right\}$, which implies that $\mathbb{P}_{p}[\operatorname{LR}(a n, 2 b n)] \leq C \cdot \mathbb{P}_{p}[\operatorname{LR}(a n, b n)]$.

By (3.1), for all $p \in[0,1], n \geq 1$, and $s \geq 0$,

$$
\mathbb{P}_{p}\left[\operatorname{LR}\left(2^{s} a n, 2^{s} b n\right)\right] \leq\left(C^{2} \mathbb{P}_{p}[\operatorname{LR}(a n, b n)]\right)^{2^{s}} .
$$

If $\liminf _{n \rightarrow \infty} p(a n, b n)=0$, then there exists $n \in \mathbb{N}$ such that $C^{2} p(a n, b n)<1$. Since the crossing probability $\mathbb{P}_{p}[\operatorname{LR}(a n, b n)]$ is continuous in $p$, there also exists $p>p_{c}$ such that $C^{2} \mathbb{P}_{p}[\operatorname{LR}(a n, b n)]<1$. For this choice of parameters, $\lim _{s \rightarrow \infty} \mathbb{P}_{p}\left[\operatorname{LR}\left(2^{s} a n, 2^{s} b n\right)\right]=0$, which is impossible, since for every $p>p_{c}$, this limit equals to 1 (see e.g. [6, Theorem 8.97]). Thus, $\liminf _{n \rightarrow \infty} p(a n, b n)>0$.

\section{Glueing}

In this section we recall a new technique for glueing paths from [5]. It will be used to adapt some arguments from planar percolation to slabs. We begin with a classical combinatorial lemma about local modifications; see e.g. [5, Lemma 7].

Lemma 4.1. Let $n \geq 1$ and $p \in(0,1)$. Let $A, B \subseteq\{0,1\}^{n}$ and $\mathbf{P}_{p}$ a product measure on $\{0,1\}^{n}$ with parameter $p$, i.e.,

$$
\mathbf{P}_{p}[\omega]=\prod_{i=1}^{n} p^{\omega_{i}}(1-p)^{1-\omega_{i}}, \quad \omega \in\{0,1\}^{n} .
$$

If there exists a map $f: A \rightarrow B$ such that for every $\omega^{\prime} \in B$, there exists a set $S \subseteq\{1, \ldots, n\}$ such that $|S| \leq s$ and

$$
\omega_{i}=\omega_{i}^{\prime}, \quad \text { for all } i \notin S \text { and } \omega \in f^{-1}\left(\omega^{\prime}\right),
$$

then

$$
\mathbf{P}_{p}[A] \leq\left(\frac{2}{\min (p, 1-p)}\right)^{s} \cdot \mathbf{P}_{p}[B] .
$$

We will often apply Lemma 4.1 in the case $p=p_{c}$ and $s$ being not bigger than the number of edges in $\overline{[-3,3]^{2}}$. Therefore, we define

$$
C_{*}=\left(\frac{2}{\min (p, 1-p)}\right)^{d \cdot 7^{2} \cdot k^{d-2}}, \quad c_{*}=\frac{1}{1+3 C_{*}} .
$$

The following lemma is essentially proven in [5, Lemma 6].

Lemma 4.2. Let $X_{1}, X_{2}, Y_{1}$, and $Y_{2}$ be disjoint connected subsets of the interior vertex boundary of $[0, m) \times[0, n)$ arranged in a counter-clockwise order. Then

$\mathbb{P}\left[\overline{X_{1}}\right.$ is connected to $\overline{X_{2}}$ in $\left.B(m, n)\right] \geq c_{*} \cdot \mathbb{P}\left[\begin{array}{l}\overline{X_{1}} \text { is connected to } \overline{Y_{1}} \text { in } B(m, n), \\ \overline{X_{2}} \text { is connected to } \overline{Y_{2}} \text { in } B(m, n)\end{array}\right]$. 
Proof. Let

$$
\begin{aligned}
& E_{i}=\left\{\overline{X_{i}} \text { is connected to } \overline{Y_{i}} \text { in } B(m, n)\right\}, \\
& X=\left\{\overline{X_{1}} \text { is connected to } \overline{X_{2}} \text { in } B(m, n)\right\} .
\end{aligned}
$$

It suffices to prove that $\mathbb{P}\left[E_{1} \cap E_{2} \cap X^{c}\right] \leq 3 C_{*} \cdot \mathbb{P}[X]$. For $i \in\{1,2\}$, consider events

$$
F_{i}=\bigcup_{z \in X_{3-i}}\left\{\bar{X}_{i} \text { is connected to } \overline{z+[-3,3]^{2}} \text { in } B(m, n)\right\} \text {. }
$$

For any $\omega \in F_{i} \cap \underline{X^{c}}$, one can choose $z \in X_{3-i}$ satisfying the requirement of $F_{i}$ so that after modifying the occupancy configuration in $\overline{z+[-3,3]^{2}}$, one obtains a configuration in which $z \times\{0\}^{d-2}$ is the unique vertex of $\overline{X_{3-i}}$ which is connected to $\overline{X_{i}}$ in $B(m, n)$. By Lemma 4.1, we obtain $\mathbb{P}\left[F_{i} \cap X^{c}\right] \leq C_{*} \cdot \mathbb{P}[X]$. Thus, it suffices to prove that $\mathbb{P}\left[E_{1} \cap E_{2} \cap F_{1}^{c} \cap F_{2}^{c}\right] \leq C_{*} \cdot \mathbb{P}[X]$. To show this, we will again use Lemma 4.1, where a suitable function $f$ : $E_{1} \cap E_{2} \cap F_{1}^{c} \cap F_{2}^{c} \rightarrow X$ will be constructed using ideas from [5, Lemma 6].

We fix an order on edges $\{e:|e|=1\}$ in $\mathbb{Z}^{d}$ and enumerate all the vertices of $\mathbb{S}$ arbitrarily. Define an order $<$ on self-avoiding paths from $\overline{X_{1}}$ to $\overline{Y_{1}}$ in $B(m, n)$ as follows. If $\gamma=\left(\gamma_{0}, \ldots, \gamma_{n}\right)$ and $\gamma^{\prime}=\left(\gamma_{0}^{\prime}, \ldots, \gamma_{n^{\prime}}^{\prime}\right)$ are two such paths, then $\gamma<\gamma^{\prime}$ if

- $\gamma_{0}$ has a smaller number than $\gamma_{0}^{\prime}$, or

- $n<n^{\prime}$ and $\gamma=\left(\gamma_{0}^{\prime}, \ldots, \gamma_{n}^{\prime}\right)$, or

- there exists $k<\min \left(n, n^{\prime}\right)$ such that $\left(\gamma_{0}, \ldots, \gamma_{k}\right)=\left(\gamma_{0}^{\prime}, \ldots, \gamma_{k}^{\prime}\right)$, and the edge $\left\{0, \gamma_{k+1}-\gamma_{k}\right\}$ is smaller than $\left\{0, \gamma_{k+1}^{\prime}-\gamma_{k}^{\prime}\right\}$.

Take $\omega \in E_{1} \cap E_{2} \cap F_{1}^{c} \cap F_{2}^{c}$. Let $\gamma_{\min }(\omega)$ be the minimal open self-avoiding path from $\overline{X_{1}}$ to $\overline{Y_{1}}$ for the above defined order. Since $\omega \in E_{2}$, there exists $z \in \gamma_{\min }(\omega)$ such that $\bar{z}$ is connected to $\overline{X_{2}}$ by an open path that does not use any edges of $\overline{\gamma_{\min }(\omega)}$. Since $\omega \in F_{1}^{c} \cap F_{2}^{c}$, for any such $z$, the set $\overline{z+[-3,3]^{2} \times\{0\}^{d-2}}$ is disjoint from $\overline{X_{1}} \cup \overline{X_{2}}$. By modifying $\omega$ in $\overline{z+[-3,3]^{2} \times\{0\}^{d-2}}$, exactly as in the proof of [5, Lemma 6, Fact 2], one obtains a new configuration $f(\omega) \in E_{1} \cap X$ such that

- $z \in \gamma_{\min }(f(\omega))$,

- $z$ is a unique vertex on $\gamma_{\min }(f(\omega))$ connected to $\overline{X_{2}}$ by an open path that does not use edges of $\gamma_{\min }(f(\omega))$,

- $\omega_{e}=f(\omega)_{e}$ for all $e \notin \overline{z+[-3,3]^{2} \times\{0\}^{d-2}}$.

This construction is tricky, but it is very carefully explained in the proof of [5, Lemma 6, Fact 2], therefore we do not repeat it here. The constructed function $f: E_{1} \cap E_{2} \cap F_{1}^{c} \cap F_{2}^{c} \rightarrow X$ satisfies the conditions of Lemma 4.1 with $s$ being the number of edges in $\overline{[-3,3]^{2}}$. Thus, $\mathbb{P}\left[E_{1} \cap E_{2} \cap F_{1}^{c} \cap F_{2}^{c}\right] \leq C_{*} \cdot \mathbb{P}\left[E_{1} \cap X\right] \leq C_{*} \cdot \mathbb{P}[X]$. The proof of the lemma is complete.

Lemma 4.2 and the FKG inequality imply the following corollary.

Corollary 4.3. Let $X_{1}, X_{2}, Y_{1}, Y_{2}$ be as in Lemma 4.2. Then

$$
\begin{aligned}
& \mathbb{P}\left[\overline{X_{1}} \text { is connected to } \overline{X_{2}} \text { in } B(m, n)\right] \\
& \quad \geq c_{*} \cdot \mathbb{P}\left[\overline{X_{1}} \text { is connected to } \overline{Y_{1}} \text { in } B(m, n)\right] \cdot \mathbb{P}\left[\overline{X_{2}} \text { is connected to } \overline{Y_{2}} \text { in } B(m, n)\right] .
\end{aligned}
$$

\section{Crossings of wide rectangles}

Proposition 5.1. If (2.1) holds for some $\rho>1$, then it holds for all $\rho>1$. 
(a)

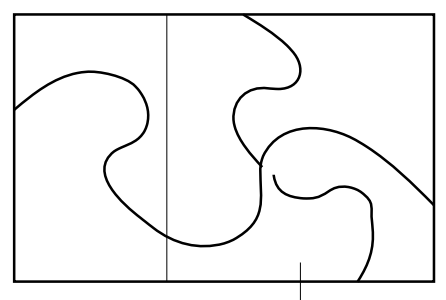

(c)

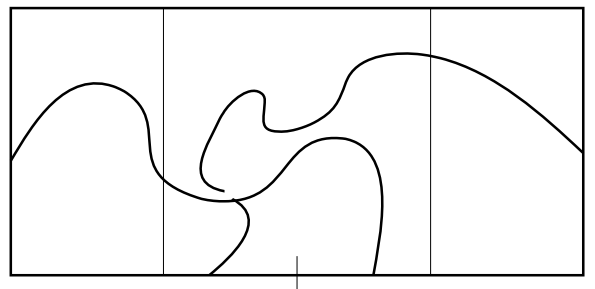

(b)

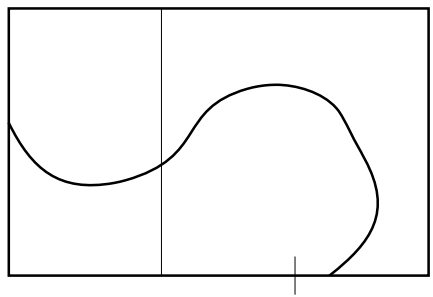

$(d)$

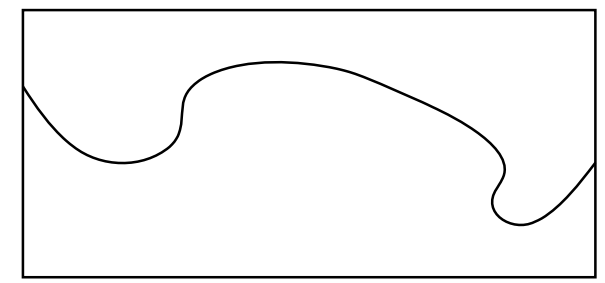

Fig. 1. (a) Left-right crossing of $B(m, n)$ and top-bottom crossing of a $\overline{[m-n, m) \times[0, n)}$ landing on the right half of the bottom, (b) path from $L(m, n)$ to $\overline{\left[m-\frac{n}{2}, m\right) \times\{0\}}$ in $B(m, n)$, (c) paths from $L(2 m-n, n)$ to $\overline{\left[m-\frac{n}{2}, m\right) \times\{0\}}$ and from $\overline{\left[m-n, m-\frac{n}{2}\right) \times\{0\}}$ to $R(2 m-n, n)$ in $B(2 m-n, n)$, (d) left-right crossing of $B(2 m-n, n)$.

Proof. This is immediate from the following inequality, which relates the crossing probability of a long rectangle with that of a shorter one. For all $m>n$,

$$
p(2 m-n, n) \geq \frac{1}{4} \cdot c_{*}^{3} \cdot p(m, n)^{4} .
$$

The inequality (5.1) follows from two applications of Corollary 4.3 illustrated on Figure 1.

\section{Crossings of rectangles: Short and long directions}

The main contribution of this paper is the following proposition, which relates the crossing probability of a rectangle in the long direction with the one in the short.

Proposition 6.1. For all $n \in \mathbb{N}$,

$$
p(44 n, 43 n) \geq \frac{c_{*}^{21} \cdot p(43 n, 44 n)^{198}}{10^{154}} .
$$

Proof. Fix $n \in \mathbb{N}$. We write

$$
B=B(43 n, 44 n), \quad L=L(43 n, 44 n), \quad R=R(43 n, 44 n),
$$

and define

$$
c=p(43 n, 44 n), \quad c^{\prime}=\frac{c_{*}{ }^{21} \cdot c^{198}}{10^{154}} .
$$

We prove the proposition by considering several cases. The first 2 steps are inspired by the ideas of Bollobás and Riordan from [2], and aimed at restricting possible shapes of left-right crossings. Steps 3 and 4 contain preliminary estimates needed to implement the main idea in Step 5.

Step 1 . We first consider the case when there is a considerable probability that a left-right crossing of $B$ stays away from the top or bottom boundary of $B$, see Figure 2. Assume that

$$
p(43 n, 42 n) \geq \frac{c}{100} .
$$




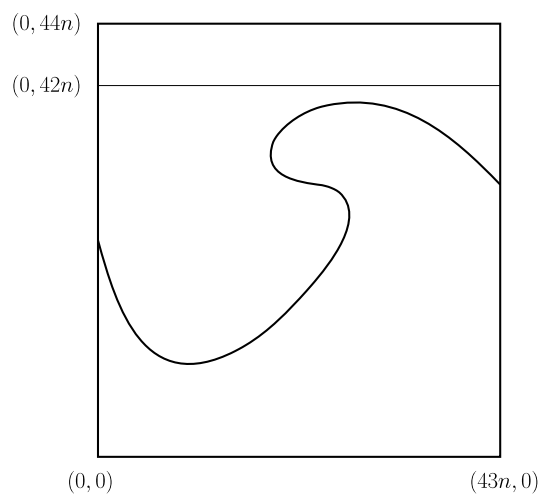

Fig. 2. Left-right crossing staying at least $2 n$ away from the top of $B(43 n, 44 n)$.

Then by (5.1),

$$
p(44 n, 43 n) \geq p(44 n, 42 n) \geq \frac{1}{4} \cdot c_{*}^{3} \cdot p(43 n, 42 n)^{4} \geq c^{\prime},
$$

which implies (6.1). Thus, we may assume that

$$
p(43 n, 42 n)<\frac{c}{100} .
$$

Step 2. Next, we consider the case when there is a considerable probability that a left-right crossing of $B$ starts sufficiently far away from the middle of $L$. Let

$$
S=\overline{\{0\} \times[20 n, 24 n)}
$$

be in the middle of $L$. Assume that

$$
\mathbb{P}[L \backslash S \text { is connected to } R \text { in } B] \geq \frac{c}{10} .
$$

Then, by reflectional symmetry,

$$
\mathbb{P}[\overline{\{0\} \times[24 n, 44 n)} \text { is connected to } R \text { in } B] \geq \frac{c}{20} .
$$

By assumption (6.2),

$$
\mathbb{P}[\overline{\{0\} \times[24 n, 44 n)} \text { is connected to } \overline{[0,43 n) \times\{2 n\}} \text { in } B] \geq \frac{c}{20}-\frac{c}{100} \geq \frac{c}{100} .
$$

By rotational symmetry, the above inequality states precisely that

$$
\mathbb{P}[\overline{\{0\} \times[0,43 n)} \text { is connected to } \overline{[22 n, 42 n) \times\{0\}} \text { in } B(42 n, 43 n)] \geq \frac{c}{100} .
$$

Similarly to the second application of Corollary 4.3 in the proof of (5.1), see Figure 3, one gets

$$
p(44 n, 43 n) \geq c_{*} \cdot\left(\frac{c}{100}\right)^{2} \geq c^{\prime},
$$

which is precisely (6.1).

Thus, we may assume, in addition to (6.2), that

$$
\mathbb{P}[L \backslash S \text { is connected to } R \text { in } B]<\frac{c}{10} \text {. }
$$


(a)

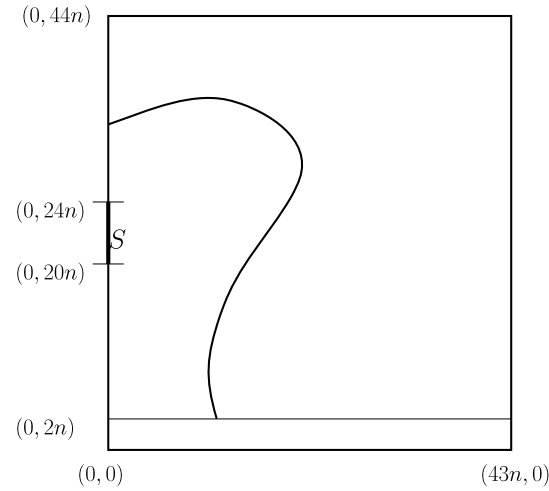

(c)

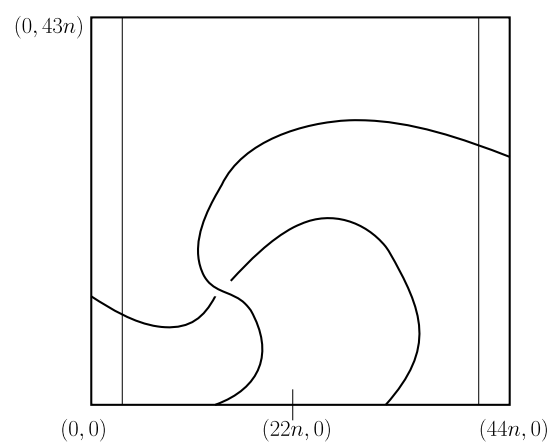

(b)

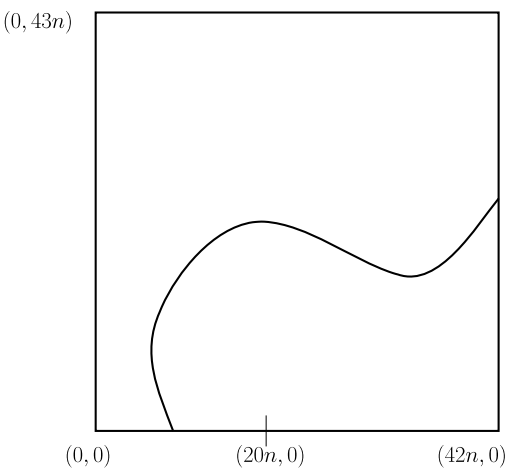

$(d)$

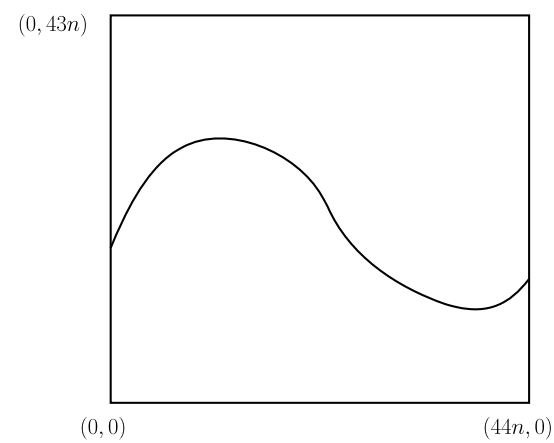

Fig. 3. (a) Part of $L$ above $S$ is connected to $\overline{[0,43 n) \times\{2 n\}}$ in $B$, (b) rotation of (a) by $\frac{\pi}{2}$, (c) $L(44 n, 43 n)$ is connected to $\overline{[22 n, 42 n) \times\{0\}}$ and $\overline{[2 n, 22 n) \times\{0\}}$ is connected to $R(44 n, 43 n)$, (d) left-right crossing of $B(44 n, 43 n)$.

Step 3. Here we consider the case when there is a considerable probability that two well-separated subsegments of $L$ are connected. For integers $a<b$, let

$$
T_{a b}=\overline{[0,43 n) \times[a, b)} \text { and } T=\overline{[0,43 n) \times \mathbb{Z}} .
$$

Assume that for some $a<b$,

$$
\mathbb{P}\left[\overline{\{0\} \times[0,4 n)} \text { is connected to } \overline{\{0\} \times[8 n, 12 n)} \text { in } T_{a b}\right] \geq \frac{c_{*} \cdot c^{18}}{10^{14}} .
$$

Then, by repetitive use of Corollary 4.3 , see Figure 4 , for each $m \geq 1$,

$$
\mathbb{P}[\overline{\{0\} \times[0,4 n)} \text { is connected to } \overline{\{0\} \times[4 n(m+1), 4 n(m+2))} \text { in } T] \geq \frac{c_{*}{ }^{2 m-1} \cdot c^{18 m}}{10^{14 m}} .
$$

Note that if $m=11$, then the event on the left hand side implies that there is a vertical crossing of $\overline{[0,43 n) \times[4 n, 48 n)}$. Thus,

$$
p(44 n, 43 n) \geq \frac{c_{*}^{21} \cdot c^{198}}{10^{154}}=c^{\prime},
$$

which gives (6.1). Therefore, we may assume, in addition to (6.2) and (6.4), that

$$
\mathbb{P}\left[\overline{\{0\} \times[0,4 n)} \text { is connected to } \overline{\{0\} \times[8 n, 12 n)} \text { in } T_{a b}\right]<\frac{c_{*} \cdot c^{18}}{10^{14}}, \quad \text { for all } a<b .
$$

Next, we derive several corollaries of assumption (6.5). 

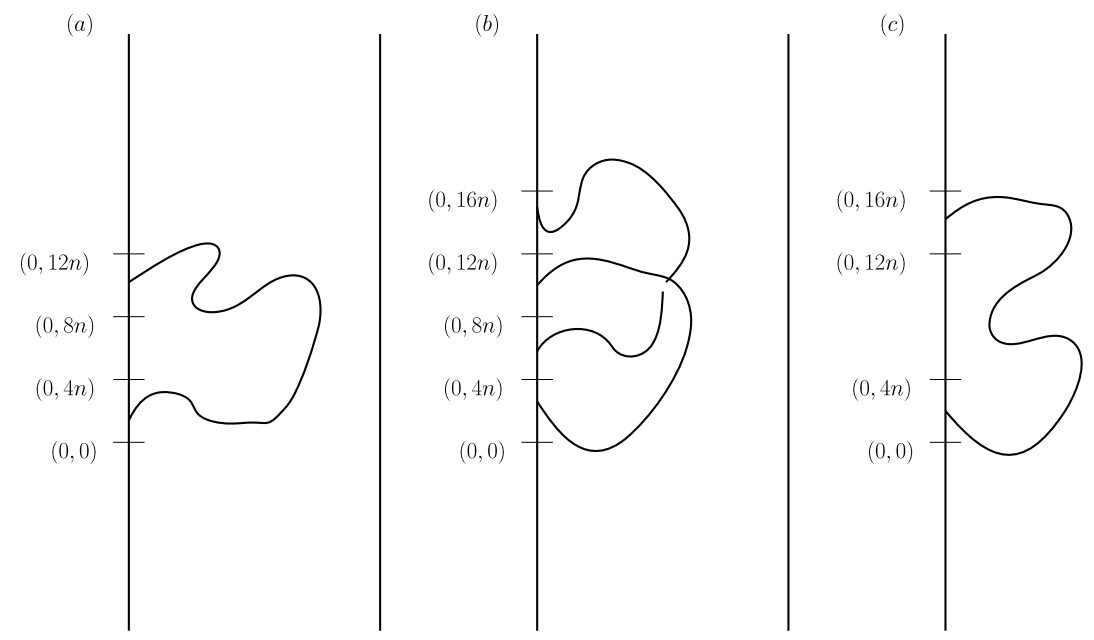

Fig. 4. Vertical extension of open paths.
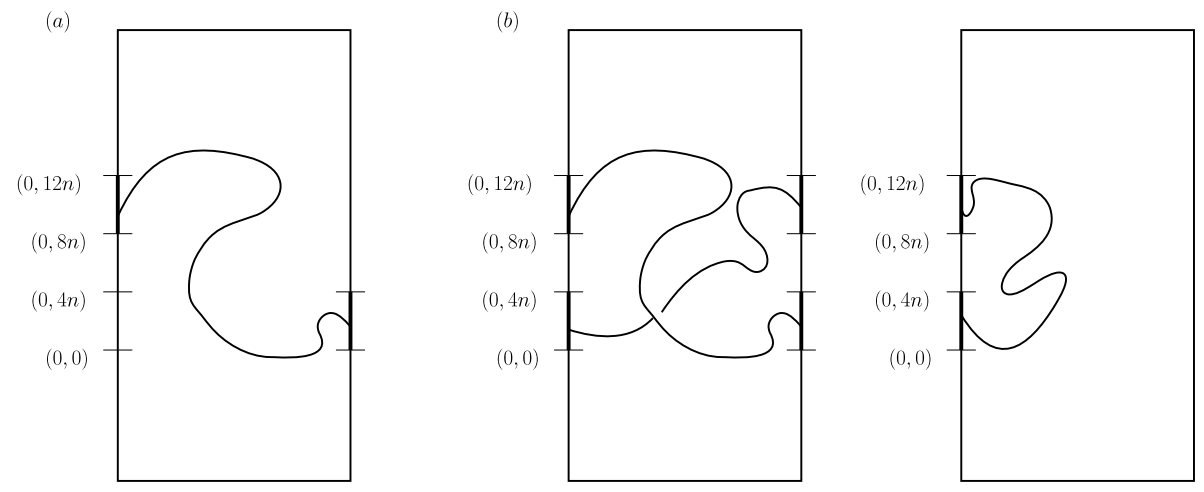

Fig. 5. (a) Illustration of the event in (6.6), (b) proof of Corollary 6.2.

Corollary 6.2. Under the assumption (6.5), for all $a<b$,

$\mathbb{P}\left[\overline{\{0\} \times[8 n, 12 n)}\right.$ is connected to $\overline{\{43 n-1\} \times[0,4 n)}$ in $\left.T_{a b}\right]<\frac{c^{9}}{10^{7}}$.

See Figure 5(a) for an illustration of the event in (6.6).

Proof. Using reflectional symmetry and Corollary 4.3 (see also Figure 5(b)),

$$
\begin{aligned}
\mathbb{P} & {\left[\overline{\{0\} \times[8 n, 12 n)} \text { is connected to } \overline{\{43 n-1\} \times[0,4 n)} \text { in } T_{a b}\right]^{2} } \\
= & \mathbb{P}\left[\overline{\{0\} \times[8 n, 12 n)} \text { is connected to } \overline{\{43 n-1\} \times[0,4 n)} \text { in } T_{a b}\right] \\
& \cdot \mathbb{P}\left[\overline{\{0\} \times[0,4 n)} \text { is connected to } \overline{\{43 n-1\} \times[8 n, 12 n)} \text { in } T_{a b}\right] \\
\leq & c_{*}{ }^{-1} \cdot \mathbb{P}\left[\overline{\{0\} \times[0,4 n)} \text { is connected to } \overline{\{0\} \times[8 n, 12 n)} \text { in } T_{a b}\right] \stackrel{(6.5)}{<} \frac{c^{18}}{10^{14}} .
\end{aligned}
$$




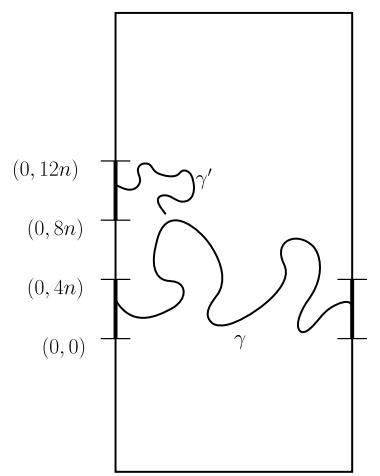

Fig. 6. An illustration of the event in (6.7).

Corollary 6.3. Under the assumption (6.5), for all $a<b$,

$$
\mathbb{P}\left[\begin{array}{c}
\text { there exist a simple path } \gamma \text { from } \overline{\{0\} \times[0,4 n)} \text { to } \overline{\{43 n-1\} \times[0,4 n)} \text { in } T_{a b} \\
\text { and a path } \gamma^{\prime} \text { from } \overline{\{0\} \times[8 n, 12 n)} \text { in } T_{a b}, \text { such that } \\
\text { the distance between } \bar{\gamma} \text { and } \overline{\gamma^{\prime}} \text { is } \leq 2
\end{array}\right]<\frac{3 \cdot c^{9}}{10^{7}} .
$$

In particular,

$$
\mathbb{P}\left[\begin{array}{c}
\text { there exist a simple path } \gamma \text { from } \overline{\{0\} \times[0,4 n)} \text { to } \overline{\{43 n-1\} \times[0,4 n)} \text { in } T \\
\text { and a path } \gamma^{\prime} \text { from } \overline{\{0\} \times[8 n, 12 n)} \text { in } T, \text { such that } \\
\text { the distance between } \bar{\gamma} \text { and } \overline{\gamma^{\prime}} \text { is } \leq 2
\end{array}\right] \leq \frac{3 \cdot c^{9}}{10^{7}} .
$$

See Figure 6 for an illustration of the event in (6.7).

Proof. It suffices to prove (6.7), as (6.8) follows from (6.7) by sending $a \rightarrow-\infty$ and $b \rightarrow+\infty$.

Denote the event in (6.7) by $A$. By the total probability formula,

$$
\begin{aligned}
\mathbb{P}[A] \leq & \mathbb{P}\left[\overline{\{0\} \times[8 n, 12 n)} \text { is connected to } \overline{\{0\} \times[0,4 n)} \text { in } T_{a b}\right] \\
& +\mathbb{P}\left[\overline{\{0\} \times[8 n, 12 n)} \text { is connected to } \overline{\{43 n-1\} \times[0,4 n)} \text { in } T_{a b}\right] \\
& +\mathbb{P}\left[\frac{A, \quad \overline{\{0\} \times[8 n, 12 n)} \text { is not connected to } \overline{\{0\} \times[0,4 n)} \text { in } T_{a b},}{\{0\} \times[8 n, 12 n)} \text { is not connected to } \overline{\{43 n-1\} \times[0,4 n)} \text { in } T_{a b}\right] .
\end{aligned}
$$

The sum of the first two probabilities is $<\frac{c_{*} \cdot c^{18}}{10^{14}}+\frac{c^{9}}{10^{7}}$, by the assumptions (6.5) and (6.6).

Denote by $A^{\prime}$ the event in the third probability. For a configuration $\omega$, let $P(\omega)$ be the set of vertices, which belong to at least one self-avoiding path from $\overline{\{0\} \times[0,4 n)}$ to $\overline{\{43 n-1\} \times[0,4 n)}$ in $T_{a b}$, one may call it a backbone. Consider a local modification map $f$ from $A^{\prime}$ to the event

$$
A^{\prime \prime}=\left\{\omega^{\prime \prime}: \begin{array}{c}
\text { there exists a unique } z\left(\omega^{\prime \prime}\right) \in P\left(\omega^{\prime \prime}\right) \text { connected to } \overline{\{0\} \times[8 n, 12 n)} \\
\text { by an open path contained in } T_{a b} \backslash P\left(\omega^{\prime \prime}\right) \text { except for the vertex } z\left(\omega^{\prime \prime}\right)
\end{array}\right\}
$$

such that for all $\omega^{\prime} \in A^{\prime}$ and all $e \notin \overline{z\left(f\left(\omega^{\prime}\right)\right)+[-3,3]^{2} \times\{0\}^{d-2}}, f\left(\omega^{\prime}\right)_{e}=\omega_{e}^{\prime}$. By Lemma 4.1, $\mathbb{P}\left[A^{\prime}\right] \leq C_{*} \cdot \mathbb{P}\left[A^{\prime \prime}\right] \leq$ $c_{*}{ }^{-1} \cdot \mathbb{P}\left[A^{\prime \prime}\right]$. Since

$$
A^{\prime \prime} \subseteq\left\{\overline{\{0\} \times[0,4 n)} \text { is connected to } \overline{\{0\} \times[8 n, 12 n)} \text { in } T_{a b}\right\},
$$

we conclude that

$$
\mathbb{P}\left[A^{\prime}\right] \leq c_{*}^{-1} \cdot \mathbb{P}\left[\overline{\{0\} \times[0,4 n)} \text { is connected to } \overline{\{0\} \times[8 n, 12 n)} \text { in } T_{a b}\right]<\frac{c^{18}}{10^{14}},
$$




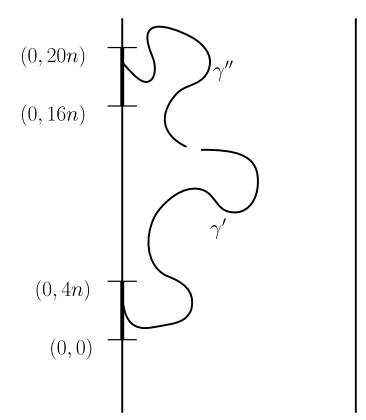

Fig. 7. An illustration of the event in (6.9).

where the last inequality follows from the assumption (6.5). Putting the bounds together,

$$
\mathbb{P}[A]<\frac{c_{*} \cdot c^{18}}{10^{14}}+\frac{c^{9}}{10^{7}}+\frac{c^{18}}{10^{14}} \leq \frac{3 \cdot c^{9}}{10^{7}} .
$$

Corollary 6.4. Under the assumptions (6.4) and (6.5),

$$
\mathbb{P}\left[\begin{array}{c}
\text { there exist a path } \gamma^{\prime} \text { from } \overline{\{0\} \times[0,4 n)} \text { in } T \\
\text { and a path } \gamma^{\prime \prime} \text { from } \overline{\{0\} \times[16 n, 20 n)} \text { in } T \text {, such that } \\
\text { the distance between } \overline{\gamma^{\prime}} \text { and } \overline{\gamma^{\prime \prime}} \text { is } \leq 4
\end{array}\right] \leq \frac{12 \cdot c^{8}}{10^{7}} .
$$

See Figure 7 for an illustration of the event in (6.9).

Proof. Denote the event in (6.9) by A. By assumption (6.4),

$$
\mathbb{P}[\overline{\{0\} \times[8 n, 12 n)} \text { is connected to } \overline{\{43 n-1\} \times[8 n, 12 n)} \text { in } T] \geq c-2 \frac{c}{10} \geq \frac{c}{2} .
$$

Since the above event and the event $A$ are increasing, by the FKG inequality,

$$
\mathbb{P}[A] \leq \frac{2}{c} \cdot \mathbb{P}[A, \overline{\{0\} \times[8 n, 12 n)} \text { is connected to } \overline{\{43 n-1\} \times[8 n, 12 n)} \text { in } T] .
$$

The intersection of the two events on the right hand side implies that for any path $\gamma$ from $\overline{\{0\} \times[8 n, 12 n)}$ to $\overline{\{43 n-1\} \times[8 n, 12 n)}$ in $T$, the distance from $\bar{\gamma}$ to $\overline{\gamma^{\prime}} \cup \overline{\gamma^{\prime \prime}}$ is $\leq 2$. Thus, by (6.8),

$$
\mathbb{P}[A] \leq \frac{2}{c} \cdot 2 \frac{3 \cdot c^{9}}{10^{7}}=\frac{12 \cdot c^{8}}{10^{7}} .
$$

Step 4. The aim of this step is to introduce a certain event of positive probability, see Proposition 6.5. Our choice of this event will be clarified in Step 5.

Recall the definition of $S$ from (6.3). For a configuration $\omega$, let $C_{S}=C_{S}(\omega)$ be the set of all $z \in T$ connected to $S$ by an open path in $T$. Let

$$
f(\omega)=\mathbb{P}\left[\overline{\{0\} \times[4 n, 8 n)} \text { is connected to } \overline{\{43 n-1\} \times \mathbb{Z}} \text { in } T \backslash \overline{C_{S}} \mid C_{S}\right](\omega),
$$

and

$$
g(\omega)=\mathbb{P}\left[\begin{array}{c}
\text { there exists a path } \gamma^{\prime} \text { from } \overline{\{0\} \times[4 n, 8 n)} \text { in } T, \text { such that } \\
\text { the distance between } \overline{\gamma^{\prime}} \text { and } \overline{C_{S}} \text { is } \leq 4
\end{array} \mid C_{S}\right](\omega) .
$$


We consider the following events:

$$
A_{1}=\{S \text { is connected to } \overline{[0,43 n) \times\{2 n\}} \text { in } T\}, \quad A_{2}=\left\{f(\omega) \geq \frac{c^{2}}{10}\right\}, \quad A_{3}=\left\{g(\omega) \leq \frac{c^{4}}{1000}\right\} .
$$

Proposition 6.5. Under the assumptions (6.2), (6.4), and (6.5),

$$
\mathbb{P}\left[A_{1} \cap A_{2} \cap A_{3}\right] \geq \frac{c^{4}}{10^{3}} .
$$

Proof. By assumptions (6.2) and (6.4),

$$
\mathbb{P}\left[A_{1}\right] \geq c-\frac{c}{10}-\frac{c}{100} \geq \frac{c}{2} .
$$

By the Markov inequality and (6.9),

$$
\mathbb{P}\left[A_{3}^{c}\right] \leq \frac{1000}{c^{4}} \cdot \mathbb{E}[g]<\frac{1000}{c^{4}} \cdot \frac{12 \cdot c^{8}}{10^{7}}=\frac{12 \cdot c^{4}}{10^{4}} .
$$

To bound $\mathbb{P}\left[A_{1} \cap A_{2}\right]$ from below we use the Paley-Zygmund inequality. Using (6.9) and the FKG inequality, we first estimate

$$
\begin{aligned}
\mathbb{E}\left[f(\omega) \cdot \mathbb{1}_{A_{1}}\right] & =\mathbb{P}\left[\frac{S \text { is connected to } \overline{[0,43 n) \times\{2 n\}} \text { in } T \text {, and }}{\{0\} \times[4 n, 8 n)} \text { is connected to } \overline{\{43 n-1\} \times \mathbb{Z}} \text { in } T \backslash \overline{C_{S}(\omega)}\right] \\
& \geq \mathbb{P}\left[\frac{S \text { is connected to } \overline{[0,43 n) \times\{2 n\}} \text { in } T, \text { and }}{\{0\} \times[4 n, 8 n)} \text { is connected to } \overline{\{43 n-1\} \times \mathbb{Z}} \text { in } T\right]-\frac{12 \cdot c^{8}}{10^{7}} \\
& \geq \mathbb{P}\left[A_{1}\right] \cdot \mathbb{P}[\overline{\{0\} \times[4 n, 8 n)} \text { is connected to } \overline{\{43 n-1\} \times \mathbb{Z}} \text { in } T]-\frac{12 \cdot c^{8}}{10^{7}} \\
& \geq \frac{c}{2}\left(c-\frac{c}{10}\right)-\frac{12 \cdot c^{8}}{10^{7}} \geq \frac{c^{2}}{5} .
\end{aligned}
$$

The Paley-Zygmund inequality for non-negative random variable $X$ states that $\mathbf{P}\left[X \geq \frac{1}{2} \mathbf{E}[X]\right] \geq \frac{1}{4} \frac{(\mathbf{E}[X])^{2}}{\mathbf{E}\left[X^{2}\right]}$. We apply it to the measure $\mathbf{P}[\cdot]=\mathbb{E}\left[\mathbb{1} \cdot \frac{\mathbb{1}_{A_{1}}}{\mathbb{P}\left[A_{1}\right]}\right]$, to get

$$
\mathbb{E}\left[\mathbb{1}_{f(\omega) \geq \frac{1}{2} \cdot \mathbb{E}\left[f(\omega) \cdot \frac{\mathbb{1}_{A_{1}}}{\mathbb{P}\left[A_{1}\right]}\right]} \cdot \frac{\mathbb{1}_{A_{1}}}{\mathbb{P}\left[A_{1}\right]}\right] \geq \frac{1}{4} \cdot\left(\mathbb{E}\left[f(\omega) \cdot \frac{\mathbb{1}_{A_{1}}}{\mathbb{P}\left[A_{1}\right]}\right]\right)^{2} .
$$

Thus,

$$
\mathbb{P}\left[A_{1} \cap A_{2}\right] \geq \frac{c^{4}}{100},
$$

and we conclude that

$$
\mathbb{P}\left[A_{1} \cap A_{2} \cap A_{3}\right] \geq \frac{c^{4}}{100}-\frac{12 \cdot c^{4}}{10^{4}} \geq \frac{c^{4}}{10^{3}} .
$$

Step 5. We are ready to conclude. For a configuration $\omega$, let $Q(\omega)$ be the set of vertices from $T$, which are connected to $S$ by an open path in $\overline{[0,43 n) \times[2 n, \infty)}$. Let $\Gamma(\omega)$ be the outer vertex boundary of $\overline{Q(\omega)}$, and $\Gamma^{\prime}(\omega)$ the mirror reflection of $\Gamma$ with respect to the hyperplane $\left\{x: x_{2}=2 n-\frac{1}{2}\right\}$. We denote the connected component of $T \backslash\left(\Gamma \cup \Gamma^{\prime}\right)$ which contains 0 by $V$. Note that $V$ is finite for any $\omega \in A_{1}$. 


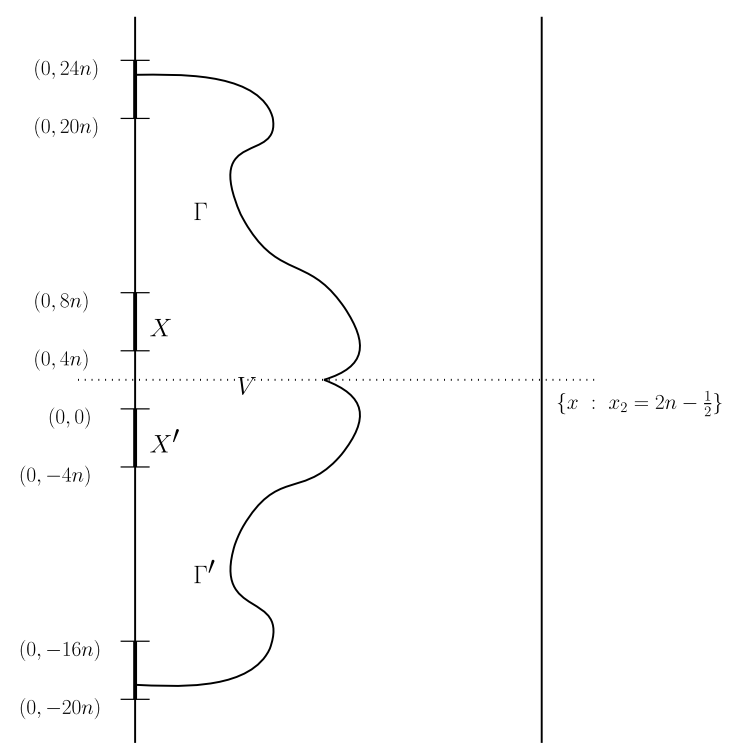

Fig. 8. An illustration of $\Gamma, \Gamma^{\prime}$, and $V$ for a configuration from the event $A_{1} \cap A_{2} \cap A_{3} . \Gamma$ is the outer vertex boundary of the cluster of $S$ in $\overline{[0,43 n) \times[2 n,+\infty)}, \Gamma^{\prime}$ is its mirror reflection with respect to the hyperplane $\left\{x: x_{2}=2 n-\frac{1}{2}\right\}$, and $V$ is the connected component of $T \backslash\left(\Gamma \cup \Gamma^{\prime}\right)$ containing the origin.

Let $X=\overline{\{0\} \times[4 n, 8 n)}$, and $X^{\prime}=\overline{\{0\} \times[-4 n, 0)}$. Note that $X^{\prime}$ is the mirror reflection of $X$ with respect to the hyperplane $\left\{x: x_{2}=2 n-\frac{1}{2}\right\}$. Moreover, if $\omega \in A_{2} \cap A_{3}$, then both $X$ and $X^{\prime}$ are contained in $V$. See Figure 8 for an illustration of all the above defined objects.

We consider an auxiliary probability space $\Omega^{\prime}$ with configurations $\omega^{\prime}$ and the same probability measure $\mathbb{P}$ on it. We compute

$\mathbb{P}\left[X\right.$ is connected to $X^{\prime}$ in $T$ by an open path in $\left.\omega^{\prime}\right]$

$\geq \mathbb{P} \otimes \mathbb{P}\left[\left(\omega, \omega^{\prime}\right):_{X}\right.$ is connected to $X^{\prime}$ in $V(\omega)$ by an open path in $\left.\omega^{\prime}\right]$

$\stackrel{(*)}{\geq} C_{*}{ }^{-1} \cdot \mathbb{P} \otimes \mathbb{P}\left[\begin{array}{c}\omega \in A_{1} \cap A_{2} \cap A_{3}, \\ X \text { is not connected to } X^{\prime} \text { in } V(\omega) \text { by an open path in } \omega^{\prime} \\ X \text { is connected to } \Gamma^{\prime}(\omega) \text { in } V(\omega) \text { by an open path in } \omega^{\prime}, \\ X^{\prime} \text { is connected to } \Gamma(\omega) \text { in } V(\omega) \text { by an open path in } \omega^{\prime}, \\ \text { there is no open path } \pi \text { in } \omega^{\prime} \text { from } X \text { in } V(\omega) \\ \text { so that the distance between } \bar{\pi} \text { and } \Gamma(\omega) \text { is } \leq 4, \\ \text { there is no open path } \pi^{\prime} \text { in } \omega^{\prime} \text { from } X^{\prime} \text { in } V(\omega) \\ \text { so that the distance between } \overline{\pi^{\prime}} \text { and } \Gamma^{\prime}(\omega) \text { is } \leq 4\end{array}\right]$

$\geq C_{*}{ }^{-1} \cdot \mathbb{E}_{\omega}\left[\mathbb{1}_{A_{1} \cap A_{2} \cap A_{3}}(\omega) \cdot \mathbb{P}_{\omega^{\prime}}\left[\begin{array}{l}X \text { is connected to } \Gamma^{\prime}(\omega) \text { in } V(\omega) \text { by an open path in } \omega^{\prime}, \\ X^{\prime} \text { is connected to } \Gamma(\omega) \text { in } V(\omega) \text { by an open path in } \omega^{\prime}\end{array}\right]\right]$

$-C_{*}{ }^{-1} \cdot \mathbb{E}_{\omega}\left[\mathbb{1}_{A_{1} \cap A_{2} \cap A_{3}}(\omega) \cdot \mathbb{P}_{\omega^{\prime}}\left[\begin{array}{c}\text { there is an open path } \pi \text { in } \omega^{\prime} \text { from } X \text { in } V(\omega) \\ \text { so that the distance between } \bar{\pi} \text { and } \Gamma(\omega) \text { is } \leq 4, \\ \text { or } \\ \text { there is an open path } \pi^{\prime} \text { in } \omega^{\prime} \text { from } X^{\prime} \text { in } V(\omega) \\ \text { so that the distance between } \overline{\pi^{\prime}} \text { and } \Gamma^{\prime}(\omega) \text { is } \leq 4\end{array}\right]\right]$

$-C_{*}{ }^{-1} \cdot \mathbb{P}\left[X\right.$ is connected to $X^{\prime}$ in $T$ by an open path in $\left.\omega^{\prime}\right]$ 


$$
\begin{aligned}
\geq & C_{*}{ }^{-1} \cdot \mathbb{E}_{\omega}\left[\mathbb{1}_{A_{1} \cap A_{2} \cap A_{3}}(\omega) \cdot\left[f(\omega)^{2}-2 g(\omega)\right]\right] \\
& -C_{*}{ }^{-1} \cdot \mathbb{P}\left[X \text { is connected to } X^{\prime} \text { in } T \text { by an open path in } \omega^{\prime}\right] .
\end{aligned}
$$

The inequality $(*)$ follows from Lemma 4.1 and a similar local transformation as in the proof of Corollary 6.3. (Mind that every path from $X$ to $\Gamma^{\prime}$ in $V$ and every path from $X^{\prime}$ to $\Gamma$ have intersecting projections, and all the "intersection points" are sufficiently far away from $\Gamma \cup \Gamma^{\prime}$ to allow for a local modification far away from $\Gamma \cup \Gamma^{\prime}$.) The last inequality comes from the FKG inequality and the definitions of event $A_{1}$ and functions $f$ and $g$.

By the definition of events $A_{2}$ and $A_{3}$ and Proposition 6.5,

$$
\mathbb{P}\left[X \text { is connected to } X^{\prime} \text { in } T\right] \geq c_{*} \cdot\left(\frac{c^{4}}{100}-2 \cdot \frac{c^{4}}{1000}\right) \cdot \frac{c^{4}}{10^{3}} .
$$

In particular, there exist $a<b$ such that

$$
\mathbb{P}\left[X \text { is connected to } X^{\prime} \text { in } T_{a b}\right] \geq \frac{c_{*} \cdot c^{8}}{10^{6}} .
$$

From this we conclude, as in the argument of Step 3, that $p(44 n, 43 n) \geq c^{\prime}$ (or simply observe that the above inequality contradicts the assumption (6.5)).

The proof of Proposition 6.1 is complete.

\section{Acknowledgements}

Bibliographic note: After this project was completed, we learned that an alternative proof of our main result was independently obtained by Newman, Tassion, and $\mathrm{Wu}$, which was subsequently written up in [8]. In the same paper, they prove that in the critical Bernoulli percolation on slabs, the probabilities of open left-right crossings of rectangles with any given aspect ratio are uniformly smaller than 1, see Remark 2.2.

\section{References}

[1] M. Aizenman. On the number of incipient spanning clusters. Nuclear Phys. B 485 (1997) 551-582. MR1431856

[2] B. Bollobas and O. Riordan. The critical probability for random Voronoi percolation in the plane is $\frac{1}{2}$. Probab. Theory Related Fields 136 (3) (2006) 417-468. MR2257131

[3] H. Duminil-Copin, C. Hongler and P. Nolin. Connection probabilities and RSW-type bounds for the two-dimensional FK Ising model. Comm. Pure Appl. Math. 64 (9) (2011) 1165-1198. MR2839298

[4] H. Duminil-Copin, V. Sidoravicius and V. Tassion. Continuity of the phase transition for planar random-cluster and Potts models with $1 \leq$ $q \leq 4$. Available at arXiv:1505.04159.

[5] H. Duminil-Copin, V. Sidoravicius and V. Tassion. Absence of infinite cluster for critical Bernoulli percolation on slabs. Comm. Pure Appl. Math. 69 (7) (2016) 1397-1411.

[6] G. R. Grimmett. Percolation. Grundlehren der mathematischen Wissenschaften 321. Springer-Verlag, Berlin, 1999. MR1707339

[7] H. Kesten. Percolation Theory for Mathematicians. Birkhäuser, Boston, MA, 1982. MR0692943

[8] C. Newman, V. Tassion and W. Wu. Critical percolation and the minimal spanning tree in slabs. Available at arXiv:1512.09107.

[9] L. Russo. A note on percolation. Z. Wahrsch. Verw. Gebiete 43 (1) (1978) 39-48. MR0488383

[10] L. Russo. On the critical percolation probabilities. Z. Wahrsch. Verw. Gebiete 56 (2) (1981) 229-237. MR0618273

[11] P. D. Seymour and D. J. A. Welsh. Percolation probabilities on the square lattice. Ann. Discrete Math. 3 (1978) 227-245. MR0494572

[12] V. Tassion. Crossing probabilities for Voronoi percolation. Ann. Probab. To appear, 2014. Available at arXiv:1410.6773. 